\title{
8
}

\section{Parameter-dependent integrals: some mathematical tools}

\author{
K. Breitung ${ }^{a}$ \\ ${ }^{a}$ Department of Civil Engineering, University of Calgary \\ 2500 University Drive N.W. \\ Calgary, Alberta, Canada, T2N 1N4
}

\section{INTRODUCTION}

In many reliability problems integrals of the following form

$$
F(\boldsymbol{\tau})=\int_{g(\boldsymbol{x}, \boldsymbol{\tau}) \leq 0} f(\boldsymbol{x}, \boldsymbol{\tau}) d \boldsymbol{x}
$$

are of interest. Here $f(\boldsymbol{x}, \tau)$ is usually a probability density and $g(\boldsymbol{x}, \tau)$ a limit state function. Both functions depend on a parameter vector $\tau$. Then $F(\tau)$ denotes the failure probability for the parameter value $\tau$.

In the case that only the integrand depends on the parameter $\tau$ the derivatives are obtained easily by differentiating under the integral sign. But the case that the limit state function depends on a parameter is of importance in reliability problems, especially in optimization. An example of such a problem is given in [7]. The concepts of asymptotic approximation methods for such integrals are outlined in [5].

\section{DERIVATIVE OF $F(\tau)$}

In [3] is was shown that under some regularity conditions the derivative $F^{\prime}(\tau)$ is given by

$F^{\prime}(\tau)=\int_{D(\tau)} f_{\tau}(\boldsymbol{x}, \tau) d \boldsymbol{x}-\int_{G(\tau)} f(\boldsymbol{y}, \tau) \frac{g_{\tau}(\boldsymbol{y}, \tau)}{|\nabla \boldsymbol{y} g(\boldsymbol{y}, \tau)|} d s_{\tau}(\boldsymbol{y})$.

Here $D(\tau)=\{\boldsymbol{y} ; g(\boldsymbol{y}, \tau)<0\}, G(\tau)=\{\boldsymbol{y} ; g(\boldsymbol{y}, \tau)=0\}$ and $d s_{\tau}(\boldsymbol{y})$ denotes surface integration over $G(\tau)$.

A disadvantage of this relation is that the second term is a surface integral, which is in general difficult to compute. Using the divergence theorem, we can transform it into a domain integral. The divergence theorem states that for a continuously differentiable vector function $\boldsymbol{u}(\boldsymbol{x})$ defined on a domain $F$ with boundary $G$ we have for the derivative 
then the following form

$\int_{F} \operatorname{div}(\boldsymbol{u}(\boldsymbol{x})) d \boldsymbol{x}=\int_{G}\langle\boldsymbol{n}(\boldsymbol{y}), \boldsymbol{u}(\boldsymbol{y})\rangle d s(\boldsymbol{y})$.

Here $\operatorname{div}(\boldsymbol{u}(\boldsymbol{x}))=\sum_{i=1}^{n} \partial u_{i}(\boldsymbol{x}) / \partial x_{i}, \boldsymbol{n}(\boldsymbol{y})$ is the outward pointing normal at $\boldsymbol{y}$ and $\langle\boldsymbol{x}, \boldsymbol{y}\rangle$ is the scalar product of $\boldsymbol{x}$ and $\boldsymbol{y}$.

First we assume that the gradient $\nabla \boldsymbol{y}(\boldsymbol{y}, \tau)$ does not vanish throughout $D(\tau)$. If we define now a vector field $\boldsymbol{u}(\boldsymbol{x})$ by

$\boldsymbol{u}(\boldsymbol{x})=\frac{f(\boldsymbol{x}, \tau) g_{\tau}(\boldsymbol{x}, \tau)}{\left|\nabla_{\boldsymbol{x}} g(\boldsymbol{x}, \tau)\right|^{2}} \nabla_{\boldsymbol{x}} g(\boldsymbol{x}, \tau)$

we get on the surface $G(\tau)$ with outward pointing normal $\boldsymbol{n}(\boldsymbol{y})=|\nabla \boldsymbol{y} g(\boldsymbol{y}, \tau)|^{-1} \nabla \boldsymbol{y} g(\boldsymbol{y}, \tau)$ for the scalar product $\langle\boldsymbol{n}(\boldsymbol{y}), \boldsymbol{u}(\boldsymbol{y})\rangle$ the form

$\langle\boldsymbol{n}(\boldsymbol{y}), \boldsymbol{u}(\boldsymbol{y})\rangle=\frac{f(\boldsymbol{x}, \tau) g_{\tau}(\boldsymbol{x}, \tau)}{\left|\nabla_{\boldsymbol{x}} g(\boldsymbol{x}, \tau)\right|}$

and therefore we have that

$\int_{G(\tau)} f(\boldsymbol{y}, \tau) \frac{g_{\tau}(\boldsymbol{y}, \tau)}{\left|\nabla_{\boldsymbol{y}} g(\boldsymbol{y}, \tau)\right|} d s_{\tau}(\boldsymbol{y})=\int_{F} \operatorname{div}\left(\frac{f(\boldsymbol{x}, \tau) g_{\tau}(\boldsymbol{x}, \tau)}{\left|\nabla_{\boldsymbol{x}} g(\boldsymbol{x}, \tau)\right|^{2}} \nabla_{\boldsymbol{x}} g(\boldsymbol{x}, \tau)\right) d \boldsymbol{x}$

This gives then for the derivative $F^{\prime}(\tau)$ the form

$F^{\prime}(\tau)=\int_{g(\boldsymbol{x}, \tau) \leq 0}\left[f_{\tau}(\boldsymbol{x}, \tau)-\operatorname{div}\left(\frac{f(\boldsymbol{x}, \tau) g_{\tau}(\boldsymbol{x}, \tau)}{\left|\nabla_{\boldsymbol{x}} g(\boldsymbol{x}, \tau)\right|^{2}} \nabla_{\boldsymbol{x}} g(\boldsymbol{x}, \tau)\right)\right] d \boldsymbol{x}$.

If now the gradient vanishes at a finite number of points in $D(\tau)$, but the Hessian $\boldsymbol{H}_{g}(\boldsymbol{x})=\left(g^{i j}(\boldsymbol{x}, \tau)\right)_{i, j=1, \ldots, n}$ is regular at all these points, equation (7) remains valid. We show this for the case of a single point $\boldsymbol{x}^{*}$ in $D(\tau)$ with vanishing gradient. We consider a domain $D^{*}(\tau)$ given by $D^{*}(\tau)=D(\tau) \backslash K(\epsilon)$, where $K(\epsilon)$ is the sphere around $\boldsymbol{x}^{*}$ with radius $\epsilon$. The value of $\epsilon$ is chosen so small that $K(\epsilon) \subset D(\tau)$. Then the divergence theorem is valid for this domain. The boundary of it are the surface $G(\tau)$ and the surface $S(\epsilon)$ of the sphere $K(\epsilon)$. So we obtain, since the outward pointing normal on $S(\epsilon)$ is $\left|\boldsymbol{x}^{*}-\boldsymbol{y}\right|^{-1}\left(\boldsymbol{x}^{*}-\boldsymbol{y}\right)$, the following equation

$$
\begin{gathered}
\int_{G(\tau)} f(\boldsymbol{y}, \tau) \frac{g_{\tau}(\boldsymbol{y}, \tau)}{|\nabla \boldsymbol{y} g(\boldsymbol{y}, \tau)|} d s_{\tau}(\boldsymbol{y})=\int_{F} \operatorname{div}\left(\frac{f(\boldsymbol{x}, \tau) g_{\tau}(\boldsymbol{x}, \tau)}{|\nabla \boldsymbol{x} g(\boldsymbol{x}, \tau)|^{2}} \nabla_{\boldsymbol{x}} g(\boldsymbol{x}, \tau)\right) d \boldsymbol{x} \\
-\underbrace{\int_{S(\epsilon)} f(\boldsymbol{y}, \tau) \frac{g_{\tau}(\boldsymbol{y}, \tau)}{|\nabla \boldsymbol{y} g(\boldsymbol{y}, \tau)|}\left\langle\frac{\nabla \boldsymbol{y} g(\boldsymbol{y}, \tau)}{\nabla \boldsymbol{y} g(\boldsymbol{y}, \tau)}, \frac{\boldsymbol{x}^{*}-\boldsymbol{y}}{\left|\boldsymbol{x}^{*}-\boldsymbol{y}\right|}\right\rangle d s_{\epsilon}(\boldsymbol{y})}_{=I(\epsilon)}
\end{gathered}
$$

with $d s_{\epsilon}(\boldsymbol{y})$ denoting surface integration over $S(\epsilon)$. 
For the last surface integral $I(\epsilon)$ we get with $K=\max \boldsymbol{y} \in K(\epsilon)\left|f(\boldsymbol{y}, \tau) g_{\tau}(\boldsymbol{y}, \tau)\right|$ the upper bound

$\left|\int_{S(\epsilon)} f(\boldsymbol{y}, \tau) \frac{g_{\tau}(\boldsymbol{y}, \tau)}{|\nabla \boldsymbol{y} g(\boldsymbol{y}, \tau)|}\left\langle\frac{\nabla \boldsymbol{y} g(\boldsymbol{y}, \tau)}{\nabla \boldsymbol{y} g(\boldsymbol{y}, \tau)}, \frac{\boldsymbol{x}^{*}-\boldsymbol{y}}{\left|\boldsymbol{x}^{*}-\boldsymbol{y}\right|}\right\rangle d s_{\epsilon}(\boldsymbol{y})\right| \leq K \int_{S(\epsilon)}|\nabla \boldsymbol{y} g(\boldsymbol{y}, \tau)|^{-1} d s_{\epsilon}(\boldsymbol{y})$.

Making a Taylor expansion of the first derivatives of $g$ at $\boldsymbol{x}^{*}$ gives

$\nabla_{\boldsymbol{y}} g(\boldsymbol{y}, \tau)=\boldsymbol{H}_{g}\left(\boldsymbol{x}^{*}\right)\left(\boldsymbol{y}-\boldsymbol{x}^{*}\right)+o(\epsilon)$.

With $\mu_{0}=\min \left|\mu_{1}\right|, \ldots,\left|\mu_{n}\right|>0$, where the $\mu_{i}$ 's are the eigenvalues of $\boldsymbol{H}_{g}\left(\boldsymbol{x}^{*}\right)$ we get for the norm

$|\nabla \boldsymbol{y} g(\boldsymbol{y}, \tau)| \geq \mu_{0}\left|\boldsymbol{y}-\boldsymbol{x}^{*}\right|+o(\epsilon)=\mu_{0} \epsilon+o(\epsilon)$.

This gives then for the integral $I(\epsilon)$ that

$|I(\epsilon)| \leq K \mu_{0} \epsilon^{-1} \int_{S(\epsilon)} d s_{\epsilon}(\boldsymbol{y})=K \mu_{0} \frac{2 \pi^{n / 2}}{\Gamma(n / 2)} \epsilon^{n-2}+o\left(\epsilon^{n-2}\right)=O\left(\epsilon^{n-2}\right)$.

Therefore equation (7) remains valid if the Hessian is regular and the dimension of the integration domain is larger than two.

\section{QUADRATIC FORMS ON SUBSPACES}

In a number of problems the definiteness of a matrix under linear constraints is of interest. Given is an $n \times n$ matrix $\boldsymbol{H}$ and a subspace $U$ spanned by $m$ linearly independent vectors $\boldsymbol{a}_{1}, \ldots, \boldsymbol{a}_{m}$. To find if the matrix is positive (or negative) on the subspace orthogonal to $U$.

We consider first the case that $\boldsymbol{a}_{i}=\boldsymbol{e}_{\boldsymbol{i}}$, i.e. the vectors $\boldsymbol{a}_{\boldsymbol{i}}$ are the first $m$ unit vectors and that $\boldsymbol{H}$ is a diagonal matrix with diagonal elements $\mu_{1}, \ldots, \mu_{n}$. Then we have for the quadratic form $\boldsymbol{x}^{T} \boldsymbol{H} \boldsymbol{x}$ the representation

$\boldsymbol{x}^{T} \boldsymbol{H} \boldsymbol{x}=\sum_{i=1}^{n} \mu_{i} x_{i}^{2}=\sum_{j=1}^{m} \mu_{j} x_{j}^{2}+\sum_{j=k+1}^{n} \mu_{j} x_{j}^{2}$.

This quadratic form is positive definite under the constraint $x_{1}=\ldots=x_{k}=0$ if the last $n-m$ diagonal elements $\mu_{m+1}, \ldots, \mu_{n}$ are positive. We consider now the quadratic form defined by

$\boldsymbol{x}^{T} \boldsymbol{H}^{*} \boldsymbol{x}=\sum_{j=1}^{k} x_{j}^{2}+\sum_{j=k+1}^{n} \mu_{j} x_{j}^{2}$.

with $\boldsymbol{H}^{*}$ a diagonal matrix with diagonal elements $1, \ldots, 1, \mu_{m+1}, \ldots, \mu_{n}$. This quadratic form is positive definite if the quadratic form $\boldsymbol{x}^{T} \boldsymbol{H} \boldsymbol{x}$ is positive definite under the constraint $x_{1}=\ldots=x_{k}=0$. The projection matrix $\boldsymbol{P}$ of projection onto the subspace spanned by $e_{1}, \ldots, e_{m}$ is given by the diagonal matrix with the first $m$ elements equal to 
one and the rest equal to zero and the projection onto the orthogonal subspace by $\boldsymbol{I}_{n}-\boldsymbol{P}$. Then we can the last equation in the form

$\boldsymbol{x}^{T} \boldsymbol{H}^{+} \boldsymbol{x}=\boldsymbol{x}^{T}\left(\boldsymbol{P P}+\left(\boldsymbol{I}_{n}-\boldsymbol{P}\right) \boldsymbol{H}\left(\boldsymbol{I}_{n}-\boldsymbol{P}\right)\right) \boldsymbol{x}$.

In the general case of a subspace spanned by $m$ arbitrary linearly independent vectors $\boldsymbol{a}_{1}, \ldots, \boldsymbol{a}_{m}$, we can reduce the problem to the case above by making a rotation such the $m$ dimensional subspace spanned by these vectors is transformed into the subspace spanned by the first $m$ new coordinate vectors. The projection matrix onto that subspace is given by

$\boldsymbol{P}=\boldsymbol{A}\left(\boldsymbol{A}^{T} \boldsymbol{A}\right)^{-1} \boldsymbol{A}^{T}$

with $\boldsymbol{A}=\left(\boldsymbol{a}_{1}, \ldots, \boldsymbol{a}_{m}\right)$. Then we get for the quadratic form $\boldsymbol{x}^{T} \boldsymbol{H}^{+} \boldsymbol{x}$ the same form as in the last equation. This gives finally: A matrix $\boldsymbol{H}$ is positive definite under the constraint $\boldsymbol{A}^{T} \boldsymbol{x}=\mathbf{o}_{m}$ iff the matrix $\boldsymbol{H}^{+}=\boldsymbol{P P}+\left(\boldsymbol{I}_{n}-\boldsymbol{P}\right) \boldsymbol{H}\left(\boldsymbol{I}_{n}-\boldsymbol{P}\right)$ is positive definite. Analogously we have that it is negative definite under these constraints iff $\boldsymbol{H}^{-}=-\boldsymbol{P P}+\left(\boldsymbol{I}_{n}-\boldsymbol{P}\right) \boldsymbol{H}\left(\boldsymbol{I}_{n}-\boldsymbol{P}\right)$. If a matrix is positive definite, can be checked easily by making a Cholesky decomposition. If this algorithm does not break down, it is positive definite.

In SORM we need the curvature correction factor for obtaining an asymptotic approximation for the failure probability. It is given in the form

$\mathrm{P}(F) \sim \Phi(-\beta) \prod_{i=1}^{n-1}\left(1-\beta \kappa_{i}\right)^{-1 / 2}$

Here the $\kappa_{i}$ 's are the main curvatures of the limit surface at the beta point $\boldsymbol{x}_{0}$ (see [2]).

In [6] it is shown that the largest eigenvalue can be obtained during the final stage of a numerical search for the beta point. The last result about quadratic forms on subspacse gives a simple method, which avoids an eigenvalue analysis for computing the main curvatures $\kappa_{1}, \ldots, \kappa_{n-1}$, for calculating this curvature correction factor $\left(\prod_{i=1}^{n-1}\left(1-\kappa_{i}\right)\right)^{-1 / 2}$.

By a rotation of the coordinates it can always be achieved that the $x_{n}$ axis is in the direction of the normal vector of the surface $G$ at $\boldsymbol{x}_{0}$ and the tangential space is spanned by the vectors in the directions of the $x_{1}, \ldots, x_{n-1}$ axes. The square of the curvature factor is then, (see [2] , eq.(25))

$$
\prod_{i=1}^{n-1}\left(1-\kappa_{i}\right)=\operatorname{det}\left(\begin{array}{cccc}
1+\frac{g_{11}\left(\boldsymbol{x}_{0}\right)}{\left|\nabla g\left(\boldsymbol{x}_{0}\right)\right|} & \frac{g_{12}\left(\boldsymbol{x}_{0}\right)}{\left|\nabla g\left(\boldsymbol{x}_{0}\right)\right|} & \ldots & \frac{g_{1, n-1}\left(\boldsymbol{x}_{0}\right)}{\left|\nabla g\left(\boldsymbol{x}_{0}\right)\right|} \\
\frac{g_{21}\left(\boldsymbol{x}_{0}\right)}{\left|\nabla g\left(\boldsymbol{x}_{0}\right)\right|} & 1+\frac{g_{22}\left(\boldsymbol{x}_{0}\right)}{\left|\nabla g\left(\boldsymbol{x}_{0}\right)\right|} & \ldots & \frac{g_{2, n-1}\left(\boldsymbol{x}_{0}\right)}{\left|\nabla g\left(\boldsymbol{x}_{0}\right)\right|} \\
\vdots & \ldots & \ddots & \vdots \\
\frac{g_{n-1,1}\left(\boldsymbol{x}_{0}\right)}{\left|\nabla g\left(\boldsymbol{x}_{0}\right)\right|} & \ldots & \ldots & 1+\frac{g_{n-1, n-1}\left(\boldsymbol{x}_{0}\right)}{\left|\nabla g\left(\boldsymbol{x}_{0}\right)\right|}
\end{array}\right)
$$

The $g_{i j}\left(\boldsymbol{x}_{0}\right)$ are the second derivatives of $g$ at $\boldsymbol{x}_{0}$ with respect to $x_{i}$ and $x_{j}$. If we add a row and a column with zeros everywhere and only 1 in the main diagonal, the value of the determinant remains the same and we obtain

$$
\operatorname{det}\left(\begin{array}{cccc}
1+\frac{g_{11}\left(\boldsymbol{x}_{0}\right)}{\left|\nabla g\left(\boldsymbol{x}_{0}\right)\right|} & \frac{g_{12}\left(\boldsymbol{x}_{0}\right)}{\left|\nabla g\left(\boldsymbol{x}_{0}\right)\right|} & \ldots & \frac{g_{1, n-1}\left(\boldsymbol{x}_{0}\right)}{\left|\nabla g\left(\boldsymbol{x}_{0}\right)\right|} \\
\frac{g_{21}\left(\boldsymbol{x}_{0}\right)}{\left|\nabla g\left(\boldsymbol{x}_{0}\right)\right|} & 1+\frac{g_{22}\left(\boldsymbol{x}_{0}\right)}{\left|\nabla g\left(\boldsymbol{x}_{0}\right)\right|} & \ldots & \frac{g_{2, n-1}\left(\boldsymbol{x}_{0}\right)}{\left|\nabla g\left(\boldsymbol{x}_{0}\right)\right|} \\
\vdots & \ldots & \ddots & \vdots \\
\frac{g_{n-1,1}\left(\boldsymbol{x}_{0}\right)}{\left|\nabla g\left(\boldsymbol{x}_{0}\right)\right|} & \ldots & \ldots & 1+\frac{g_{n-1, n-1}\left(\boldsymbol{x}_{0}\right)}{\left|\nabla g\left(\boldsymbol{x}_{0}\right)\right|}
\end{array}\right)
$$




$$
=\operatorname{det} \underbrace{\left(\begin{array}{ccccc}
1+\frac{g_{11}\left(\boldsymbol{x}_{0}\right)}{\left|\nabla g\left(\boldsymbol{x}_{0}\right)\right|} & \frac{g_{12}\left(\boldsymbol{x}_{0}\right)}{\left|\nabla g\left(\boldsymbol{x}_{0}\right)\right|} & \ldots & \frac{g_{1, n-1}\left(\boldsymbol{x}_{0}\right)}{\left|\nabla g\left(\boldsymbol{x}_{0}\right)\right|} & 0 \\
\frac{g_{21}\left(\boldsymbol{x}_{0}\right)}{\left|\nabla g\left(\boldsymbol{x}_{0}\right)\right|} & 1+\frac{g_{22}\left(\boldsymbol{x}_{0}\right)}{\left|\nabla g\left(\boldsymbol{x}_{0}\right)\right|} & \ldots & \frac{g_{2, n-1}\left(\boldsymbol{x}_{0}\right)}{\left|\nabla g\left(\boldsymbol{x}_{0}\right)\right|} & 0 \\
\vdots & \ldots & \ddots & \vdots & \vdots \\
\frac{g_{n-1,1}\left(\boldsymbol{x}_{0}\right)}{\left|\nabla g\left(\boldsymbol{x}_{0}\right)\right|} & \ldots & \ldots & 1+\frac{g_{n-1, n-1}\left(\boldsymbol{x}_{0}\right)}{\left|\nabla g\left(\boldsymbol{x}_{0}\right)\right|} & 0 \\
0 & \ldots & \ldots & 0 & 1
\end{array}\right)}_{=D} .
$$

If we set

$$
\boldsymbol{H}\left(\boldsymbol{x}_{0}\right)=\left(\delta_{i j}+\left|\nabla g\left(\boldsymbol{x}_{0}\right)\right|^{-1} g_{i j}\left(\boldsymbol{x}_{0}\right)\right)_{i, j=1, \ldots, n},
$$

we get that the matrix $\boldsymbol{D}$ can be written in the form

$D=P^{T} H\left(x_{0}\right) P+e_{n} e_{n}^{T}$

with $\boldsymbol{e}_{n}=(0, \ldots, 0,1)^{T}$ and $\boldsymbol{P}=\boldsymbol{I}_{n}-\boldsymbol{e}_{n} \boldsymbol{e}_{n}^{T}$. Due to the special choice of the coordinate system, $\boldsymbol{e}_{n}$ is the normal vector of the surface $G$ at $\boldsymbol{x}_{0}$ and $\boldsymbol{P}$ is the projection matrix onto the tangential space of $G$ at $\boldsymbol{x}_{0}$. But this formulation is invariant under linear coordinate changes; we just have to replace $\boldsymbol{e}_{n}$ by the normal vector $\boldsymbol{n}$ in the new coordinates $\boldsymbol{n}=\left|\nabla g\left(\boldsymbol{x}_{0}\right)\right|^{-1} \nabla g\left(\boldsymbol{x}_{0}\right)$. So we have for an arbitrary coordinate system the following expression for the curvature factor

$$
\prod_{i=1}^{n-1}\left(1-\kappa_{i}\right)=\operatorname{det}(\boldsymbol{D})=\operatorname{det}\left(\boldsymbol{P}^{T} \boldsymbol{H}\left(\boldsymbol{x}_{0}\right) \boldsymbol{P}+\left|\nabla g\left(\boldsymbol{x}_{0}\right)\right|^{-2} \nabla g\left(\boldsymbol{x}_{0}\right)\left(\nabla g\left(\boldsymbol{x}_{0}\right)\right)^{T}\right)
$$

with $\boldsymbol{P}=\boldsymbol{I}_{n}-\left|\nabla g\left(\boldsymbol{x}_{0}\right)\right|^{-2} \nabla g\left(\boldsymbol{x}_{0}\right)\left(\nabla g\left(\boldsymbol{x}_{0}\right)\right)^{T}$.

Since the function $|\boldsymbol{x}|^{2}$ has a local minimum at the point $\boldsymbol{x}_{0}$ under the constraint $g(\boldsymbol{x})=$ 0 the matrix $\boldsymbol{H}\left(\boldsymbol{x}_{0}\right)$ is positive definite under the linear constraint $\boldsymbol{n}^{T} \boldsymbol{x}=0$, if the extremum is regular. Therefore in this case the determinant of $D$ can be found from the Cholesky decomposition of $\boldsymbol{P}^{T} \boldsymbol{H}\left(\boldsymbol{x}_{0}\right) \boldsymbol{P}+\left|\nabla g\left(\boldsymbol{x}_{0}\right)\right|^{-2} \nabla g\left(\boldsymbol{x}_{0}\right)\left(\nabla g\left(\boldsymbol{x}_{0}\right)\right)^{T}$.

In the same way we can obtain the asymptotic approximation in the case of non-normal random variables with p.d.f. $f(\boldsymbol{x})$. Here the asymptotic approximation is given by

$\mathrm{P}(F) \sim(2 \pi)^{(n-1) / 2} \frac{f\left(\boldsymbol{x}^{*}\right)}{\left|\nabla l\left(\boldsymbol{x}^{*}\right)\right|\left|\operatorname{det}\left(\boldsymbol{H}^{*}\left(\boldsymbol{x}^{*}\right)\right)\right|^{1 / 2}}$

with $l(\boldsymbol{x})=\ln (f(\boldsymbol{x}))$ the $\log$-likelihood function (see [4]). The matrix $\boldsymbol{H}^{*}\left(\boldsymbol{x}^{*}\right)$ is defined by

$$
\left.\boldsymbol{H}^{*}\left(\boldsymbol{x}^{*}\right)\right)=\boldsymbol{A}^{T} \boldsymbol{H}\left(\boldsymbol{x}^{*}\right) \boldsymbol{A} .
$$

Here $\boldsymbol{H}=\left(l^{i j}\left(\boldsymbol{x}^{*}\right)-\left|\nabla l\left(\boldsymbol{x}^{*}\right)\right|\left|\nabla g\left(\boldsymbol{x}^{*}\right)\right|^{-1} g^{i j}\left(\boldsymbol{x}^{*}\right)\right)_{i, j=1, \ldots, n}$ and $\boldsymbol{A}=\left(\boldsymbol{a}_{1}, \ldots, \boldsymbol{a}_{n-1}\right)$, where the $\boldsymbol{a}_{i}$ 's form an orthonormal basis of the tangential space of the limit state surface at $\boldsymbol{x}^{*}$.

If the log-likelihood function has a regular maximum with respect to the failure domain at $\boldsymbol{x}^{*}$, then we can compute $\operatorname{det}\left(\boldsymbol{H}^{*}\left(\boldsymbol{x}^{*}\right)\right)$ again as

$$
\operatorname{det}\left(\boldsymbol{H}^{*}\left(\boldsymbol{x}^{*}\right)\right)=\operatorname{det}\left(\boldsymbol{P} \boldsymbol{H}\left(\boldsymbol{x}^{*}\right) \boldsymbol{P}-\boldsymbol{n}\left(\boldsymbol{x}^{*}\right) \boldsymbol{n}\left(\boldsymbol{x}^{*}\right)^{T}\right) .
$$

Here $\boldsymbol{P}=\boldsymbol{I}_{n}-\boldsymbol{n}\left(\boldsymbol{x}^{*}\right) \boldsymbol{n}\left(\boldsymbol{x}^{*}\right)^{T}$. 


\section{PARAMETER DEPENDENCE OF THE PML-POINT}

We consider the problem that the reliability problem is a function of a parameter $\tau$. Then the point of maximal likelihood (PML) depends on this parameter value. The change of the beta value in FORM theory under parameter changes was treated in [1]. We consider an integral as in equation (1), but with only a scalar parameter $\tau$.

Following from the results of the last paragraph the failure probability is approximated making a Taylor expansion of the log-likelihood function around the PML and using the Laplace method. We asssume that for all feasible values of $\tau$ there is exactly one PML $\boldsymbol{x}^{*}=\boldsymbol{x}^{*}(\tau)$ on the limit state surface $G(\tau)$.

The gradients of these functions with respect to the first $n$ variables $x_{1}, \ldots, x_{n}$ are denoted by $\nabla_{\boldsymbol{x}} f$ (resp. $\nabla_{\boldsymbol{x}} g$ ) and the partial derivative with respect to $\tau$ by $f_{\tau}$ (resp. $\left.g_{\tau}\right)$. The Hessian of a function $f(\boldsymbol{x}, \tau)$ with respect to the first $n$ variables is written as $\boldsymbol{H}_{f}$.

We assume that for a fixed value of $\tau$ there is a unique PML $\boldsymbol{x}^{*}(\tau)$ and we write in a shorthand notation $\boldsymbol{x}^{*}$. The vector of the first derivatives of $\boldsymbol{x}^{*}$ with respect to $\tau$ is written as $\boldsymbol{x}_{\tau}^{*}$. This point is a stationary point of the Lagrangian function $L(\boldsymbol{x}, \lambda, \tau)$ defined by

$L=f-\lambda g$.

Therefore for the point $\boldsymbol{x}^{*}(\tau)$ the following equation system

$$
\begin{aligned}
\nabla_{\boldsymbol{x}} f-\lambda \nabla_{\boldsymbol{x}} g & =\mathbf{o}_{n}, \\
g & =0 .
\end{aligned}
$$

must be fulfilled.

To find the derivatives of the coordinates of the PML with respect to parameter changes, we differentiate this system with respect to $\tau$ and set all derivatives equal to zero. This gives then

$$
\begin{aligned}
\boldsymbol{H}_{f} \boldsymbol{x}_{\tau}^{*}+\nabla_{\boldsymbol{x}} f_{\tau}-\lambda_{\tau} \nabla_{\boldsymbol{x}} g-\lambda\left(\boldsymbol{H}_{g} \boldsymbol{x}_{\tau}^{*}+\nabla_{\boldsymbol{x}} g_{\tau}\right) & =\mathbf{o}_{n}, \\
\left\langle\nabla_{\boldsymbol{x}} g, \boldsymbol{x}_{\tau}^{*}\right\rangle+g_{\tau} & =0 .
\end{aligned}
$$

Rearranging the terms gives

$$
\begin{aligned}
\left(\boldsymbol{H}_{f}-\lambda \boldsymbol{H}_{g}\right) \boldsymbol{x}_{\tau}^{*}+\frac{\partial}{\partial \tau}\left(\nabla_{\boldsymbol{x}} f-\lambda \nabla_{\boldsymbol{x}} g\right) & =\lambda_{\tau} \nabla_{\boldsymbol{x}} g \\
\left\langle\nabla_{\boldsymbol{x}} g, \boldsymbol{x}_{\tau}^{*}\right\rangle & =-g_{\tau} .
\end{aligned}
$$

Since always $\nabla_{\boldsymbol{x}} f-\lambda \nabla_{\boldsymbol{x}} g=\mathbf{o}_{n}$, we get deleting this term

$$
\begin{aligned}
\left(\boldsymbol{H}_{f}-\lambda \boldsymbol{H}_{g}\right) \boldsymbol{x}_{\tau}^{*} & =\lambda_{\tau} \nabla_{\boldsymbol{x}} g \\
\left.\left\langle\nabla_{\boldsymbol{x}} g\right), \boldsymbol{x}_{\tau}^{*}\right\rangle & =-g_{\tau} .
\end{aligned}
$$

This gives then for the vector $\boldsymbol{x}_{\tau}^{*}$ the form

$$
\boldsymbol{x}_{\tau}^{*}=\lambda_{\tau}\left(\boldsymbol{H}_{f}-\lambda \boldsymbol{H}_{g}\right)^{-1} \nabla_{\boldsymbol{x}} g \text {. }
$$

To determine the value of $\lambda_{\tau}$ we compute the scalar product of $\boldsymbol{x}_{\tau}^{*}$ and $\nabla_{\boldsymbol{x}} g$, yielding

$$
\left\langle\nabla_{\boldsymbol{x}} g, \boldsymbol{x}_{\tau}^{*}\right\rangle=\lambda_{\tau}\left(\nabla_{\boldsymbol{x}} g\right)^{T}\left(\boldsymbol{H}_{f}-\lambda \boldsymbol{H}_{g}\right)^{-1} \nabla_{\boldsymbol{x}} g .
$$


From equation (29) follows then that the lefthand side is equal to $-g_{\tau}$ and so we get

$g_{\tau}=-\lambda_{\tau}\left(\nabla_{\boldsymbol{x}} g\right)^{T}\left(\boldsymbol{H}_{f}-\lambda \boldsymbol{H}_{g}\right)^{-1} \nabla_{\boldsymbol{x}} g$.

This gives then for the derivative $\lambda_{\tau}$ then

$\lambda_{\tau}=-\left[\left(\nabla_{\boldsymbol{x}} g\right)^{T}\left(\boldsymbol{H}_{f}-\lambda \boldsymbol{H}_{g}\right)^{-1} \nabla_{\boldsymbol{x}} g\right]^{-1} g_{\tau}$

Inserted into equation (30) we obtain

$\boldsymbol{x}_{\tau}^{*}=-\frac{g_{\tau}}{\left(\nabla_{\boldsymbol{x}} g\right)^{T}\left(\boldsymbol{H}_{f}-\lambda \boldsymbol{H}_{g}\right)^{-1} \nabla_{\boldsymbol{x}} g}\left(\boldsymbol{H}_{f}+\lambda \boldsymbol{H}_{g}\right)^{-1} \nabla_{\boldsymbol{x}} g$.

The change of the value $f^{*}=f\left(\boldsymbol{x}^{*}(\tau), \tau\right)$ is given by

$f_{\tau}^{*}=\left\langle\nabla_{\boldsymbol{x}} f, \boldsymbol{x}_{\tau}^{*}\right\rangle+f_{\tau}$.

Due to the Lagrange multiplier theorem, we can replace the gradient of $f$ by the gradient of $g$, giving

$f_{\tau}^{*}=\lambda\left\langle\nabla_{\boldsymbol{x}} g, \boldsymbol{x}_{\tau}^{*}\right\rangle+f_{\tau}$.

From equation (29) we get then

$f_{\tau}^{*}=-\lambda g_{\tau}+f_{\tau}$.

If $f_{\tau}=0$, the Lagrange multiplier $\lambda$ gives the change of the value of $f^{*}$ relative to the negative change of $g$ with respect to $\tau$. If the constraint is given in the form $g(\boldsymbol{x})-\tau=0$ and the p.d.f. depends not on $\tau$, we obtain the simple form

$f_{\tau}^{*}=\lambda$.

If we consider the approximation for the failure probability given in equation (22) and we neglect the change of the quantities in the denominator we get approximately

$\frac{\partial \mathrm{P}(F)}{\partial \tau} \approx \frac{(2 \pi)^{-(n-1) / 2}}{\left|\nabla l\left(\boldsymbol{x}^{*}\right)\right|\left|\operatorname{det}\left(\tilde{\boldsymbol{H}}\left(\boldsymbol{x}^{*}\right)\right)\right|^{1 / 2}}\left(-\lambda g_{\tau}+f_{\tau}\right)$.

\section{References}

[1] P. Bjerager and S. Krenk. Parametric sensitivity in first order reliability theory. Journal of the Engineering Mechanics Division ASCE, 115:1577-1582, 1989.

[2] K. Breitung. Asymptotic approximations for multinormal integrals. Journal of the Engineering Mechanics Division ASCE, 110(3):357-366, 1984.

[3] K. Breitung. Parameter sensitivity of failure probabilities. In A. Der-Kiureghian and P. Thoft-Christensen, editors, Reliability and Optimization of Structural Systems '90, Proceedings of the 3rd IFIP WG 7.5 Conference, Berkeley, California, pages 43-51, New York, 1991. Springer. Lecture Notes in Engineering 61. 
[4] K. Breitung. Probability approximations by log likelihood maximization. Journal of the Engineering Mechanics Division ASCE, 117(3):457-477, 1991.

[5] K. Breitung. Asymptotic Approximations for Probability Integrals. Springer, Berlin, 1994. Lecture Notes in Mathematics, Nr. 1592.

[6] A. Der Kiureghian and M. De Stefano. Efficient algorithm for second-order reliability analysis. Journal of the Engineering Mechanics Division ASCE, 117(12):2904-2923, 1991.

[7] A. Der Kiureghian, Y. Zhung, and Ch.-Ch. Li. Inverse reliability problem. Journal of the Engineering Mechanics Division ASCE, 120(5):1154-1159, 1994. 